\title{
Same same but different. Die Feldtheorien Fligsteins und Bourdieus und das Potenzial einer wechselseitig informierten Perspektive für die Wirtschaftssoziologie
}

\author{
Lisa Suckert
}

Online publiziert: 27. Juni 2018

(C) Der/die Autor(en) 2018

Zusammenfassung Feldtheoretische Zugänge haben in der Wirtschaftssoziologie Konjunktur. So wird dem Konzept Feld bereits das Potenzial zugesprochen, das hegemoniale Paradigma der Einbettung abzulösen. Der Beitrag arbeitet den erkenntnistheoretischen Mehrwert eines solchen feldtheoretischen Zugangs heraus, indem er die Ansätze Neil Fligsteins und Pierre Bourdieus systematisch miteinander ins Gespräch bringt. Entlang ihrer Entstehungskontexte, theoretischer und methodologischer Bezugspunkte, Untersuchungsinteressen und zentraler Konzepte werden Gemeinsamkeiten, aber auch Unterschiede beider Ansätze herausgearbeitet. Die aus diesem Vergleich resultierenden Erkenntnisse bilden die Basis einer feldtheoretischen und im Kern relationalen Perspektive auf ökonomische Phänomene, die neue Impulse für die Wirtschaftssoziologie bereit hält.

Schlüsselwörter Feldtheorie · Bourdieu · Fligstein · Wirtschaftssoziologie · Relationale Soziologie

\section{Same same but different. The field theoretical approaches of Fligstein and Bourdieu and the potential of a mutually informed perspective for economic sociology}

\begin{abstract}
Field theoretical approaches have become popular within economic sociology. Many even consider the notion of ,field“ a suitable alternative to the hegemonic paradigm of ,embeddedness“. This article outlines the epistemological potential of a field theoretical perspective by systematically comparing the approaches of Neil Fligstein and Pierre Bourdieu. By examining their respective contexts of emergence, their theoretical and methodological references, their research interests and their cen-
\end{abstract}

L. Suckert $(\bowtie)$

Max-Planck-Institut für Gesellschaftsforschung, Paulstraße 3, 50676 Köln, Deutschland

E-Mail: suckert@mpifg.de 
tral concepts, both similarities and differences between the two approaches become visible. The resulting insights allow for a field theoretical and profoundly relational perspective on economic phenomena that might stimulate ongoing debates within economic sociology.

Keywords Field theory $\cdot$ Bourdieu $\cdot$ Fligstein $\cdot$ Economic sociology $\cdot$ Relational sociology

\section{Du pareil au même ? La théorie des champs de Fligstein et de Bourdieu et les potentialités de leur dialogue mutuel en sociologie économique}

Résumé Les approches en termes de théorie des champs ont la cote en sociologie économique. On prête ainsi déjà au concept de champ la capacité de détrôner le paradigme hégémonique de l'encastrement. Cet article met en évidence l'intérêt épistémologique de ce type d'approche en faisant dialoguer point par point les approches de Neil Fligstein et Pierre Bourdieu. Les points communs mais aussi les différences entre ces deux approches sont mises en évidence à travers le contexte de leur élaboration, leurs références théoriques et méthodologiques, leurs intérêts de connaissance et leurs principaux concepts. Les conclusions tirées de cette comparaison constituent la base d'une perspective essentiellement relationnelle sur les phénomènes économiques en termes de champs offrant de nouvelles impulsions à la sociologie économique.

Mots-clés Théorie des champs · Bourdieu · Fligstein · Sociologie économique · Sociologie relationnelle

\section{Einleitung}

Seit ihrer Herausbildung ist die Neue Wirtschaftssoziologie (NWS) ein zentraler Schauplatz der relationalen Soziologie im Sinne einer netzwerkstrukturellen Perspektive. Wichtige Gründungsväter ${ }^{1}$ der amerikanischen NWS wie Mark Granovetter, Ronald Burt oder Harrison White haben zugleich bedeutsame Beiträge zur Entwicklung der sozialen Netzwerkanalyse geleistet. Das für die NWS wegweisende Paradigma einer ,eingebetteten“ Wirtschaft wurde nicht zuletzt entlang der Netzwerkmetapher und der damit implizierten Interaktionsbeziehungen entwickelt.

Die zweite große Spielart der relationalen Soziologie stellen feldtheoretische Zugänge dar. Auch diese sind in der Wirtschaftssoziologie nicht unbekannt. Ganz im Gegenteil lässt sich eine gewisse wirtschaftssoziologische Euphorie für das Konzept des Feldes feststellen, die am besten in der Einschätzung von Richard Swedberg (2009, S. 79) - seinerseits ein Gründungsvater der NWS - zum Ausdruck kommt: ,[...] die einzige bis dato existierende Theoriealternative in der Wirtschaftssoziolo-

\footnotetext{
1 Wie so oft lässt sich auch im Rahmen der NWS nur schwerlich von „Gründungsmüttern“ sprechen. Wirtschaftssoziologinnen, die einen relationalen Ansatz in den Mittelpunkt ihrer Arbeit stellen sind z.B. Sophie Mützel (2009), Nina Bandelj und Danica Purg (2006) oder neuerdings Viviana Zelizer (2012).
} 
gie zum Konzept der Einbettung [sei ...] das Verständnis von Wirtschaft als Feld, mit allem was dieser Begriff impliziert." Die Sympathiebekundungen, die sie feldtheoretischen Zugängen entgegenbringt, hat die NWS jedoch bisher nur zögerlich in ihre wirtschaftssoziologische Forschungspraxis übersetzt. Empirische Studien, die die Feldtheorie als umfassenden Analyserahmen heranziehen, sind im Bereich der NWS nach wie vor selten. Allzu oft bleibt es bei oberflächlichen Zitaten, unverbindlichen Verweisen oder dem selektiven Herausgreifen einzelner Konzepte.

Auch der vorliegende Aufsatz nutzt die Feldtheorie nicht, um wirtschaftssoziologische Fragen im Rahmen einer empirischen Studie zu beantworten (vgl. hierzu Suckert 2015, 2017). Er zielt jedoch darauf, die wirtschaftssoziologische Erklärungskraft feldtheoretischer Zugänge systematisch greifbar zu machen, indem er zwei Ansätze, denen innerhalb der NWS besonders viel Potenzial zugeschrieben wird, miteinander ins Gespräch bringt: die Feldtheorien von Pierre Bourdieu und Neil Fligstein ${ }^{2}$. Die folgende Gegenüberstellung beider Ansätze soll nicht nur ihre Konturen, Gemeinsamkeiten und Differenzen hervortreten lassen, sondern auch Gelegenheit bieten, sie kritisch auf ihren wirtschaftssoziologischen Mehrwert zu befragen. Grundlegend ist dabei die Beobachtung, dass beide Feldtheorien ,same same but different" sind: Ausgehend von der Metapher des Feldes ähnelt sich ihr grundlegender, relationaler Zugriff auf soziale Phänomene. Gleichzeitig bestechen sie durch je unterschiedliche Blickwinkel - und weisen damit auch verschiedene blinde Flecken für die wirtschaftssoziologische Analyse auf. Es wird daher für eine wechselseitig informierte Perspektive plädiert, die es erlaubt, die jeweiligen Schwächen zu kompensieren, um so wirtschaftliche Phänomene in größerer empirischer Breite betrachten zu können.

In den folgenden beiden Abschnitten wird zunächst das Untersuchungsinteresse der NWS rekapituliert, um davon ausgehend Grundlinien einer feldtheoretischen Perspektive auf die Wirtschaft zu skizzieren. Während hier die Gemeinsamkeiten beider Ansätze herausgearbeitet werden, adressiert der dritte Abschnitt die Unterschiede zwischen Bourdieus und Fligsteins Perspektive. Entlang ihrer Entstehungskontexte und Untersuchungsinteressen werden die jeweiligen Eigenarten beider Ansätze herausgestellt. So werden erkenntnistheoretische Potenziale, aber auch Desiderate gleichermaßen sichtbar. Daran anschließend wird diskutiert, inwiefern sich beide Feldtheorien integrieren lassen und worin der Mehrwert einer wechselseitig informierten Perspektive bestünde. Das Fazit fasst die wichtigsten Implikationen für ein feldtheoretisches wirtschaftssoziologisches Forschungsprogramm zusammen.

\section{Das Untersuchungsinteresse der Neuen Wirtschaftssoziologie}

Möchte man das erkenntnistheoretische Potenzial feldtheoretischer Zugänge für die NWS aufzeigen, kommt man nicht umhin zu bestimmen, worin deren eigentliches Forschungsinteresse besteht. Kaum jemand hat das Anliegen der NWS, die sich

\footnotetext{
2 Ausgehend vom Fokus dieses Beitrags wird vorrangig auf die Arbeiten des Wirtschaftssoziologen Neil Fligstein verwiesen, obgleich dieser seinen Ansatz zusammen mit Doug McAdam (s.u.) entwickelt hat.
} 
Mitte der 1980er Jahre in den USA formierte, so pointiert beschrieben wie Marion Fourcade (2013, S. 37): „Wirtschaftssoziologie [ist] der Teil der Soziologie [...], die sich mit den Objekten der Ökonomie statt mit den ökonomischen Objekten im eigentlichen Sinne beschäftigt." [Hervorh. u. gramm. Fehler i. O.] Demzufolge stehen weder die klassischen wirtschaftssoziologischen Arbeiten von z.B. Max Weber, Émile Durkheim oder Georg Simmel im Mittelpunkt der NWS, noch geht es ihr bloß um eine anders gelagerte Erklärung wirtschaftlicher Phänomene per se. Stattdessen nutzt sie soziologische Theorien, um die Mängel klassischer ökonomischer Zugänge aufzuzeigen, ${ }^{3}$ und versucht, einen Gegenpol zu den als neoklassisch verstandenen Wirtschaftswissenschaften zu etablieren. Die NWS definiert sich also wesentlich über ihre Antagonisten.

Dabei wird vor allem kritisiert, dass Ökonomen das reibungslose Gelingen ökonomischer Koordination als ,natürlich“ missverstehen. Für die NWS ist das Zustandekommen von Märkten, Unternehmen oder Konsum hingegen nicht selbstverständlich, sondern an voraussetzungsvolle soziale Konstruktions- und Strukturierungsprozesse gebunden. Ökonomische Koordination wird als problematisch betrachtet, da sie unablässig von Unsicherheiten geprägt und bedroht ist: Woher sollen Akteure wissen, wie viel ein Produkt, eine Dienstleistung oder die eigene Arbeitskraft wert sind, und wie können sie sichergehen, dass andere diese Einschätzung teilen? Warum vertrauen Akteure darauf, nicht ständig übervorteilt zu werden? Wie können sie legitimes Profitstreben von unlauteren ökonomischen Strategien unterscheiden? Aus der Perspektive der NWS müsste anhaltende Unsicherheit ökonomische Koordination nahezu unmöglich machen (Beckert 2007; Deutschmann 2007). Im Kern geht es ihr somit darum zu verstehen, wie beständiges ökonomisches Handeln trotz Unsicherheit möglich ist: Unter welchen sozialen Voraussetzungen findet es statt? Durch welche sozialen Prozesse und Strukturen wird es ermöglicht - und dabei gleichzeitig begrenzt? Diese Fragen werden von den neoklassischen Wirtschaftswissenschaften bislang ausgeblendet.

Die vielfältigen Antworten, die die NWS hingegen vorgelegt hat, werden im Anschluss an Mark Granovetters (1985) wegweisenden Aufsatz gemeinhin unter den Begriff der „Einbettung“ subsumiert. Wirtschaft kann demnach nicht als Produkt atomistisch agierender homines oeconomici verstanden werden, sondern muss als soziales Phänomen mit dem Instrumentarium der Soziologie erschlossen werden. Wie oben angedeutet, konzipierten Granovetter und andere Gründungsväter der NWS Einbettung allerdings zunächst rein netzwerkartig. Das Paradigma der Einbettung, das im Zentrum des wirtschaftssoziologischen Selbstverständnisses steht, erscheint damit zwar als relational (Fourcade 2013), bezieht sich jedoch lediglich auf Relationen im Sinne von Interaktionsbeziehungen und Netzwerkpositionen. Diese Verkürzung wurde vielfach kritisiert, entfalte das Soziale doch nicht ausschließlich in Form von Netzwerkbeziehungen seine Wirkmächtigkeit (Zukin und DiMaggio 1990, S. 3). Als Reaktion auf diese Kritik identifizierte die NWS sukzessive weitere „Formen der Einbettung“. Neben Netzwerken gelten ihr heute vor allem Kultur

\footnotetext{
3 Dieses Motiv findet sich auch bei den genannten klassischen Wirtschaftssoziologen - die NWS ignoriert diese Tradition jedoch.
} 
und Institutionen als die zentralen sozialen Mechanismen, über die Unsicherheit reduziert und ökonomisches Handeln ermöglicht wird (Dobbin 2004, S. 4).

Die enorme Ausweitung, die das Einbettungskonzept damit über die Zeit erfahren hat, entkräftet nun zwar die Kritik an einem rein netzwerkstrukturellen Zugang und immunisiert den Ansatz geradezu - ob seiner schieren Breite - gegen Beanstandungen aller Art, was die Etablierung eines neuen, von der Einbettungssemantik klar abgrenzbaren Paradigmas in der Wirtschaftssoziologie schwer möglich macht. Doch gleichzeitig hat „Einbettung“ durch diese Ausweitung erheblich an Aussagekraft verloren (Krippner und Alvarez 2007).

In diesem Zusammenhang wird feldtheoretischen Zugängen immer wieder das Potenzial zugeschrieben, die Vorherrschaft der Einbettungsansätze herauszufordern (Beckert 2010; Swedberg 2009, S. 79). Aufgrund unübersehbarer Schnittmengen mit bestehenden kulturellen oder institutionellen Einbettungskonzepten stellen sie zwar kein „neues“ Paradigma im engeren Sinne dar. Gleichwohl bieten feldtheoretische Zugänge eine Perspektive, die über bestehende Ansätze in der NWS hinausgeht und vor allem durch eines besticht: Ein divergierendes Verständnis von Relationen, das soziale Wirkungsverhältnisse auch jenseits von interaktionistischen Netzwerkbeziehungen fassbar macht. Das Potenzial einer solchen Perspektive für die Forschungsagenda der NWS wird im Folgenden herausgestellt.

\section{Same same: Grundlagen einer feldtheoretischen Perspektive auf die Wirtschaft}

Den Kern feldtheoretischer Zugänge bildet die Metapher des Feldes, die auf multiple Assoziationen verweist. Eine zentrale Referenz stellen naturwissenschaftliche Feldtheorien dar. Semantik und Konzepte, die zur Beschreibung elektromagnetischer Phänomene und einhergehender Kräfte gebräuchlich waren, wurden maßgeblich von Kurt Lewin (1939) als Heuristik in die Sozialwissenschaften übertragen (Martin 2003; Müller 2014, S. 73). Sowohl Bourdieu als auch Fligstein ${ }^{4}$ verweisen jedoch zusätzlich auf die Assoziation des Spielfeldes (Bourdieu und Wacquant 1996, S. 127; Fligstein und McAdam 2012a, S. 31). Auf diesem Feld wetteifern Gegner in einem regelgebundenen Spiel um Vorteile und den Sieg. Ausgehend von diesen beiden zentralen Deutungen der Feldmetapher ${ }^{5}$ werden im Folgenden drei grundlegende Facetten bzw. Gemeinsamkeiten der Ansätze Bourdieus und Fligsteins herausgearbeitet. Dabei zeigt sich der zutiefst relationale Charakter feldtheoretischer Zugänge (vgl. Bernhard und Schmidt-Wellenburg 2012, S. $48 \mathrm{f}$.).

1) Soziale Kraftfelder und relative Feldgrenzen. Die Elemente in elektromagnetischen Feldern werden weder durch starre Gitterstrukturen noch durch eine äußere Membran zusammengehalten, sondern von unsichtbaren inneren Kräften, die auf die

\footnotetext{
${ }^{4}$ Einen weiteren wichtigen, aber hier nicht betrachteten wirtschaftssoziologischen Zugang stellen ,organizational fields" dar, die im organisationssoziologischen Neo-Institutionalismus prominent sind. Für eine Gegenüberstellung vgl. Fligstein und Kluttz (2016) sowie Florian (2008).

5 Vgl. auch Witte (2014, S. 48 ff.), der für den Bourdieu'schen Ansatz nochmals zwischen Spiel- und Kampffeldern unterscheidet.
} 
einzelnen Elemente wirken und sie dadurch zueinander in Beziehung setzen. Ganz ähnlich geht auch die soziologische Feldtheorie davon aus, dass Akteure in sozialen Feldern nicht per se durch manifeste Netzwerkbeziehungen oder fixe Außengrenzen im Sinne formaler Institutionen gebunden sind. Auch hier ist eine innere, auf den ersten Blick unsichtbare Kraft am Werk, die sich am besten als die der geteilten Wahrnehmungsstrukturen beschreiben lässt. Diese beinhalten zum einen den Glauben an die Sinnhaftigkeit des Spiels und dessen, ,was auf dem Spiel steht“ (Fligstein und McAdam 2012a, S. 10), der von Bourdieu auch als ,illusio“ (1998a, S. 140f.) bezeichnet wird. Darüber hinaus teilen Feldakteure aber auch Wissen über Spielregeln und -strategien. Sie wissen, wie das Spiel, an dem sie teilnehmen, funktioniert, was die relevanten Trümpfe sind, welche Taktiken möglich sind, welches Handeln sanktioniert wird oder zum Sieg verhilft. Durch geteilte Wahrnehmungsstrukturen gelingt es den Feldakteuren, das Handeln anderer sinnvoll zu interpretieren und zu ihrem eigenen Möglichkeitshorizont in Beziehung zu setzen.

2) Relationen zwischen Feldelementen. Die Feldmetapher lenkt den Fokus zudem auf jene Kräfte, die zwischen den einzelnen Feldelementen wirken und damit einen Raum aufspannen. Die „,sozialen Kräfteverhältnisse“, für die sich soziologische Feldtheorien interessieren, lassen sich als Machtverhältnisse verstehen. Macht kann dabei auf den unterschiedlichsten „Trümpfen“ beruhen. Deren Wert ist jedoch nicht absolut messbar. Erst in Relation zur Ressourcenausstattung aller anderen Feldakteure und zu den Regeln des Feldes werden sie zu Stärken und Schwächen, die Macht verleihen oder entziehen. Der soziale Raum der Positionen, für den sich die Bourdieu'sche wie die Fligstein'sche Feldtheorie interessiert, ist somit ein Raum der ungleichen Machtpositionen: Darin können ,challenger“ und ,incumbents“ (Fligstein), „Dominante“ und „Dominierte“ (Bourdieu) verortet werden.

3) Relationen zwischen Feldern. Magnetische Felder verfügen wie gesagt nicht über starre Grenzen, sondern über Feldkräfte, die vom Zentrum zur Peripherie hin abnehmen. Folglich können sich Felder und einhergehende Feldeffekte überlagern. Auch die betrachteten soziologischen Feldtheorien gehen davon aus, dass soziale Felder in gegenseitigen Wechselverhältnissen stehen. Sie werden als eingebettet in ein „System von Feldern“ (Fligstein) bzw. ein „Feld der Macht“ (Bourdieu) verstanden ${ }^{6}$. Felder können sich z. B. gegenseitig bedingen, indem ein Feld auf die Regeln des anderen Einfluss nimmt oder ihm relevante Ressourcen bereitstellt. Ähnlich wie die Elemente in einem Feld können auch Felder aufeinander wirken und um Vorherrschaft ringen. Sowohl die Bourdieu'sche als auch die Fligstein'sche Feldtheorie gehen davon aus, dass sich die Funktionsweise sozialer Felder nur verstehen lässt, wenn die Relationen zwischen Feldern in die Analyse einbezogen werden.

Beide Ansätze offenbaren somit eine Reihe von Gemeinsamkeiten, die sich entlang der Metapher des Feldes fassen lassen. Es wird deutlich, dass beide Theorieansätze das Soziale als relational skizzieren. Anders als herkömmliche Einbettungsansätze, wie sie der NWS zugrunde liegen, stellen Bourdieu und Fligstein jedoch nicht primär auf manifeste, persönliche Interaktionsbeziehungen ab. Von Interesse ist vielmehr, wie sich Akteure, Ressourcen und Felder zueinander ins Verhältnis setzen lassen. Nicht der direkte Kontakt ist ausschlaggebend, sondern das, was

${ }^{6}$ Zum „Feld der Macht“ vgl. auch Schmitz, Witte und Gengnagel (2016). 
Bourdieu und Wacquant als „objektive Relationen“ (1996, S. 125 f.) beschreiben: latente, wechselseitig aufeinander bezogene Positionen, die in einem sozialen Raum gedacht werden. Doch welche Perspektive auf wirtschaftliche Phänomene impliziert ein derartiger feldtheoretischer Zugang und wie ist es um die Anschlussfähigkeit an die bestehende wirtschaftssoziologische Forschung bestellt? Ausgehend von den oben abgebildeten Facetten zeigt sich, dass eine feldtheoretische Perspektive auf die Wirtschaft 1) ein Interesse an kognitiven Phänomenen in der Ökonomie, 2) ein Interesse an Ungleichheit, Macht und Konflikten in der Ökonomie und 3) ein Interesse am Zusammenspiel ökonomischer Felder jenseits der Kluft zwischen Mikro- und Makro-Ansätzen impliziert.

1) Interesse an kognitiven Phänomenen in der Ökonomie. Der Fokus auf geteilte Wahrnehmungsstrukturen, die soziale Felder gleich einer unsichtbaren Kraft zusammenhalten und gegenüber anderen Feldern abgrenzen, impliziert eine Wirtschaftssoziologie, die sich maßgeblich für ideelle und kognitive Phänomene interessiert. Eine feldtheoretische Perspektive auf die Wirtschaft nimmt demnach das Zustandekommen von geteilten Bewertungsmaßstäben, d.h. jenen Kategorien, denen im Feld Wert zugesprochen wird, in den Blick. Damit ergeben sich nicht nur markante Schnittstellen zur Soziologie der Bewertung (Lamont 2012), sondern auch zu etablierten wirtschaftssoziologischen Forschungssträngen, die sich mit der Rolle von (informellen) Institutionen und Kultur in ökonomischen Bewertungsprozessen auseinandersetzen (im deutschsprachigen Raum z.B. Aspers 2007; Beckert und Rössel 2004; Diaz-Bone 2007) - und sich damit ebenso wie die Feldtheorie von rein netzwerkstrukturellen Erklärungsmodellen abgrenzen. Zentral ist jedoch, dass sich eine feldtheoretische Perspektive nicht mit der inhaltlichen Analyse geteilter Wahrnehmungs- und Bewertungsstrukturen begnügt, sondern auf deren umkämpfte Natur abstellt. Im Fokus steht, wie diese kognitiven Muster zustande kommen, d.h. wie sie in anhaltenden Auseinandersetzungen geformt, transformiert und als Machtinstrumente eingesetzt werden.

2) Interesse an Ungleichheit, Macht und Konflikten in der Ökonomie. Eine zweite Besonderheit einer feldtheoretischen Perspektive auf die Wirtschaft stellt somit der Fokus auf Kämpfe, Konflikte und Machtgefälle dar. Abweichend von wirtschaftswissenschaftlichen Annahmen ist ökonomischer Wettbewerb nicht nur als ein Ringen um Profitchancen zu verstehen, sondern als ein umfassender, anhaltender Kampf, der sich auf verschiedenartige Ressourcen, aber auch auf die Interpretation der Spielregeln bezieht. Hier eröffnen sich für die NWS neue Sichtweisen: Institutionen, Netzwerke und kulturelle Rahmungen reduzieren zwar Unsicherheit und ermöglichen damit ökonomisches Handeln, sie sind aber nicht funktionalistisch, sondern primär als Resultate vorausgegangener Kämpfe und der dazugehörigen Machtkonstellationen zu verstehen. Eine feldtheoretische Perspektive erlaubt es, stärker in den Blick zu nehmen wie z. B. branchenspezifische Standards, Marktaufsichtsbehörden, Best Practices oder Quasi-Kartelle entstehen und welche Machtinteressen dabei eine Rolle spielen. Im Zentrum dieser Sichtweise steht ein relationales Verständnis von Ökonomie, das nicht Relationen im Sinne von manifesten Interaktionsbeziehungen, sondern objektive Relationen im Sinne wechselseitiger Positionen im sozialen Raum betont (vgl. Bourdieu und Wacquant 1996, S. 125). Der feldtheoretische Zugang verweist damit nicht auf Netzwerke, sondern auf ökonomische Ungleichheit - ein 
wichtiger und klassischer Themenbereich, der in der NWS bislang kaum Beachtung gefunden hat. $^{7}$

3) Interesse am Zusammenspiel ökonomischer Felder. Die Neue Wirtschaftssoziologie versteht sich maßgeblich als Marktsoziologie. Als solche operiert sie auf der Meso-Ebene und nimmt zumeist Branchen, d.h. Konkurrenzbeziehungen zwischen Anbietern in den Blick. Bezüge zur Organisationssoziologie, die das Geschehen auf Unternehmensebene beleuchtet, und insbesondere zur Politischen Ökonomie, die das Verhältnis von (internationalen) Industrien und Volkswirtschaften analysiert, werden nur selten hergestellt. Auch Pierre Bourdieu und Neil Fligstein haben zumeist Gruppen von Anbietern ins Zentrum ihrer empirischen Wirtschaftsanalysen gestellt (z. B. Fligstein 1990; Bourdieu 1998b). Nichtsdestotrotz erlaubt es die feldtheoretische Perspektive, das Zusammenspiel verschiedener ökonomischer Felder und Ebenen zu betrachten. So können nicht nur Branchen, sondern auch Unternehmen und deren Abteilungen, Volkswirtschaften und sogar die globale Ökonomie insgesamt jeweils als ökonomische Felder mit spezifischen Regeln, spezifischen Positionen und spezifischen Kämpfen betrachtet werden. Das Ineinandergreifen dieser Felder und ihre gegenseitige Bedingtheit erlauben ein umfassenderes Verständnis von Ökonomie, das jenseits der in der Wirtschaftssoziologie meist üblichen Aufteilung in Mikro- und Makro-Ebenen agiert. Zudem erlaubt es eine feldtheoretische Perspektive, auch den Einfluss nicht-ökonomischer Felder stärker zu berücksichtigen. Sowohl Bourdieu als auch Fligstein zeigen immer wieder auf, wie insbesondere der Staat (der auch als Feld zu verstehen ist) erheblichen Einfluss auf ökonomische Felder nimmt, indem er z.B. Produktstandards vorschreibt, Mitarbeiter schult oder durch Subventionen die Nachfrage ankurbelt. Eine feldtheoretische Perspektive bietet das Instrumentarium, um diese Interdependenzen auch konzeptionell abzubilden.

In der Zusammenschau zeigt sich, dass die Ansätze von Neil Fligstein und Pierre Bourdieu wichtige Gemeinsamkeiten teilen, die als Kern einer feldtheoretischen Perspektive bezeichnet werden können. Eine dezidiert feldtheoretische Betrachtung ökonomischer Zusammenhänge zeichnet sich demnach durch ein verstärktes Interesse an kognitiven Phänomenen, an Macht und Konflikten sowie am Zusammenspiel verschiedener (ökonomischer) Felder aus. Die erkenntnistheoretischen Potenziale und Anschlussmöglichkeiten, die diese Perspektive und ihr Fokus auf objektive Relationen für die Wirtschaftssoziologie bieten, wurden aufgezeigt. Jenseits dieses Kerns werden die beiden Ansätze jedoch von einer Reihe unterschiedlicher Standpunkte geprägt: Sie sind zwar ,same same“, d.h. in ihrer grundlegenden Ausrichtung sehr ähnlich, aber dennoch verschieden. Diese Unterschiede werden im nächsten Abschnitt herausgearbeitet.

\footnotetext{
7 Gleichwohl können Netzwerkkontakte im Sinne einer Ressource in die Analyse dieser Machtgefälle einbezogen werden (Bernhard 2010; Mützel 2006).
} 


\section{But different: Zur Abgrenzung der Fligstein'schen und Bourdieu'schen Perspektive}

Wie alle kognitiven Rahmungen, entstehen auch wissenschaftliche Theorien niemals in einem sozialen Vakuum. Die akademische Sozialisation und das theoretische Erbe der Wissenschaftler, die sie entwerfen, sowie die Spezifika der Untersuchungsgegenstände, entlang derer sie entwickelt werden, aber auch die Methodologie, die genutzt wird, um Theorie und Empirie ins Gespräch zu bringen, prägen Theorien nachhaltig. Daher sollen in einem ersten Schritt die verschiedenen Entstehungskontexte und Bezugspunkte sowohl der Bourdieu'schen als auch der Fligstein'schen Feldtheorie näher beleuchtet werden. Daran anschließend werden die resultierenden unterschiedlichen Untersuchungsinteressen nochmals entlang sechs zentraler Facetten aufgeschlüsselt: Gegenübergestellt werden die unterschiedliche Bedeutung, die Akteurshandeln, Historizität und Wandel jeweils zugewiesen wird, die Fähigkeit beider Theorien, Kooperation und Machtpositionen konzeptionell greifbar zu machen, sowie das kritische Potenzial beider Ansätze.

\subsection{Entstehungskontexte: Theoretische und methodologische Bezugspunkte}

Das herausragende Erklärungspotenzial der Bourdieu'schen Feldtheorie ist nicht zuletzt darauf zurückzuführen, dass das Konzept des Feldes untrennbar mit zwei weiteren Konzepten, Habitus und Kapital, verbunden ist. Dieser Dreiklang bildet den Kern von Bourdieus Perspektive und wurde im Laufe seiner über 50-jährigen wissenschaftlichen Tätigkeit an den unterschiedlichsten empirischen Gegenständen weiterentwickelt und geschärft. Den Ausgangspunkt bilden Bourdieus Studien im Algerien der 1950er Jahre. Aus der Not heraus, seinen Militärdienst in Algerien ableisten zu müssen, widmete sich Bourdieu einer ethnologischen Studie der Kabylen. Anhand dieses Stammes zeigte er den dramatischen Wandel der algerischen Gesellschaft unter dem Einfluss des kapitalistischen Kolonialismus auf. Es waren diese Jahre des Feldstudiums, die den Philosophiestudenten und Absolventen der École Normale zu einem überzeugten Soziologen werden ließen. Die Grundmotive der Bourdieu'schen Perspektive - der Fokus auf Machtkämpfe und insbesondere das Aufdecken von verborgenen Formen symbolischer Herrschaft, die Prägekraft inkorporierter Geschichte oder die Bedeutung von alltäglichen, unhinterfragten Praktiken - sind bereits in diesen Studien angelegt (Schultheis 2007, S. 10f.). Die theoretische Systematisierung erfolgt später, z.B. in Entwurf einer Theorie der Praxis von 1972 (1976) oder Sozialer Sinn von 1980 (1987). Zeitlebens hat Bourdieu seine theoretischen Konzepte als Werkzeuge für die empirische Forschung betrachtet, die es Forschern ermöglichen, neuartige Fragen an die empirische Realität zu stellen. Trotz der bemerkenswerten Kontinuität der Bourdieu'schen Theoriearbeiten (Müller 2014, S. 21) wurden seine feldtheoretischen Konzepte daher immer wieder an verschiedenste empirische Gegenstände angepasst.

Die außergewöhnliche Vielfalt seiner Forschung und insbesondere sein Interesse an kultureller Produktion haben dazu geführt, dass Bourdieu, zum eigenen Missfallen (1998b, S. 163), lange nicht als Wirtschaftssoziologie wahrgenommen wurde. Dabei setzt er sich auch in seinen zentralen kultursoziologischen Werken, wie z.B. Die 
feinen Unterschiede (2007) oder Die Regeln der Kunst (1999), mit ökonomischen Sachverhalten auseinander. Darüber hinaus gibt es eine Reihe von Arbeiten, die sich dezidiert wirtschaftlicher Untersuchungsgegenstände annehmen (1990, 1994, 1998b, 2000; Bourdieu et al. 1963; Bourdieu und Boltanski 1975). Auch hier wird das grundlegende Interesse seines feldtheoretischen Zugangs an Macht, sozialer Distinktion und der Reproduktion sozialer Ungleichheiten deutlich.

Bourdieus Ansatz ist, nicht zuletzt durch seine Primärsozialisation als Philosoph und seine ethnographischen Arbeiten, von vielfältigen theoretischen Bezugspunkten geprägt und zeichnet sich durch den Versuch aus, gängige soziologische Dualismen zu überwinden (1987, S. 49). So finden sich in seinen Konzepten einerseits Einflüsse des französischen Strukturalismus (v.a. Lévi-Strauss und Althusser), die sich etwa darin zeigen, dass objektiven sozialen Strukturen (und weniger dyadischen Interaktionen) erhebliche Prägekraft zugesprochen wird. Andererseits entwickelt Bourdieu seine Feldtheorie entlang einer phänomenologischen Denkweise, die er u.a. von Husserl, Mauss und Weber übernimmt. Das Interesse an objektiven, aber latenten Strukturen verbindet sich so mit der genauen Analyse von Alltagspraktiken, körperlichen Fähigkeiten und Phänomenen des „Nicht-Ausgesprochenen“, sowie der Art und Weise wie Menschen alledem subjektive Bedeutung verleihen.

Dieser doppelte strukturalistisch-objektivistische und phänomenologisch-subjektivistische Zugang wird durch die methodologische Prägung Bourdieus gestärkt: Einerseits ist seine Methode der Feldanalyse von Anfang an durch ethnographisches Arbeiten geprägt, was ihm den Zugang zu Alltagswahrnehmungen erlaubte. Andererseits machte sich Bourdieu in seinen Algerienstudien und der einhergehenden Zusammenarbeit mit dem INSEE $^{8}$ mit statistischen Analysemethoden vertraut, die abstraktere gesellschaftliche Strukturen abbilden. Hervorzuheben ist hier insbesondere die von Benzécri (1982) entwickelte ,analyse des donnés“ (im Deutschen v.a. als Korrespondenzanalyse bekannt), die die Bourdieu'sche Idee des Feldkonzepts und die zugrundeliegende räumliche Vorstellung von Gesellschaft maßgeblich beeinflusst hat (Blasius und Schmitz 2013; Lebaron 2009): „Und wenn ich im allgemeinen lieber mit der Korrespondenzanalyse arbeite als zum Beispiel mit der multiple regression, dann eben auch deshalb, weil sie eine relationale Technik der Datenanalyse darstellt, deren Philosophie genau dem entspricht, was in meinen Augen die Realität der sozialen Welt ausmacht. Es ist eine Technik, die in Relationen ,denkt", genau wie ich das mit dem Begriff Feld versuche“" (Bourdieu und Wacquant 1996, S. 125 f.). Dieser empirische, theoretische und methodologische Entstehungskontext der Bourdieu'schen Perspektive bedingt eine Reihe von Eigenheiten, die sie von einer Feldtheorie Fligstein'scher Prägung abgrenzen.

Neil Fligstein entwickelte seine Feldtheorie, die er selbst als ,theory of strategic action fields" bezeichnet, in den 1980er Jahren in Zusammenarbeit mit Doug McAdam an der University of Arizona. Während McAdam sich mit sozialen Bewegungen und insbesondere der amerikanischen Bürgerrechtsbewegung beschäftigte (McAdam 1990), etablierte sich Fligstein zu dieser Zeit als Wirtschafts- und Organisationssoziologe. Er arbeitete an einem seiner Hauptwerke, The transformation of corporate control (1990), das die historischen Strategien großer US-Konzerne und insbeson-

\footnotetext{
8 Institut national de la statistique et des études économiques.
} 
dere deren aufkommende Finanzorientierung untersucht. Anhand dieser Studie entwickelte Fligstein den Begriff des „Kontrollkonzepts“, das geteilte Wahrnehmungsstrukturen umschreibt. Kontrollkonzepte stabilisieren Felder, sind aber fortwährend umkämpft. Betrachtet man die empirischen Arbeiten von sowohl McAdam als auch Fligstein, so fällt auf, dass sich beide primär für Umbrüche (von z.B. Industrien, Zivilgesellschaft) interessieren und diese über einen längeren Zeitraum verfolgen. Der charakteristische theoretische Fokus auf Wandel (s.u.) entspringt somit auch hier den empirischen Interessen der beiden Forscher.

Während beide Autoren in den 1990er Jahren ihre Karrieren in unterschiedlichen sozialwissenschaftlichen Bereichen verfolgten, wurden lediglich einzelne Versatzstücke ihrer Theorie veröffentlicht (Fligstein 1997; Fligstein und Stone Sweet 2002; McAdam 1999). Eine wirklich systematische, gemeinsame Aufarbeitung der Theorie erfolgte erst Anfang dieses Jahrzehnts (Fligstein und McAdam 2011, 2012b). In ihrem Buch A theory of fields (Fligstein und McAdam 2012a) bekräftigen die beiden Autoren, eine ,general theory of social change and stability“, d.h. eine Großtheorie mit Allgemeingültigkeitsanspruch vorgelegt zu haben. Eben dies hatte Bourdieu, ausgehend von der festen Überzeugung, dass theoretische Konzepte erst in konkreten empirischen Kontexten ihre Bedeutung entfalten, stets für seinen umfassend(er)en theoretischen Ansatz abgelehnt. In der Tat zeigt sich, dass der Fligstein'sche Feldbegriff, trotz der postulierten Allgemeingültigkeit, stark an der wirtschaftssoziologischen Forschung seines Autors orientiert ist. Im Zentrum stehen hier ökonomische und politische Felder, in denen Wettbewerb offen und bewusst ausgetragen wird. Diesem Theorieansatz wird daher, in Abgrenzung zu Bourdieu, vorgeworfen, für verborgene Machtkämpfe und rein symbolische Herrschaft blind zu sein (Medvetz 2013). Für die wirtschaftssoziologischen Anwendungsmöglichkeiten, die hier in den Blick genommen werden sollen, ist diese konzeptionelle Engführung jedoch nicht nur von Nachteil: Anders als das Bourdieu'sche Instrumentarium ist die Fligstein'sche Feldtheorie von Haus aus auf ökonomische Sachverhalte zugeschnitten. Auch wenn Fligstein seinen Zugang keinesfalls als Rational-Choice-Ansatz missverstanden wissen möchte (Kluttz und Fligstein 2016, S. 187), ermöglicht es dieser, offenen Wettbewerb und bewusst-reflektierte Strategien problemlos konzeptionell zu fassen.

Zentrale theoretische Bezugspunkte sind, wenig überraschend, die Arbeiten Pierre Bourdieus, die McAdam und Fligstein als „closest to our perspective“ (Fligstein und McAdam 2012a, S. 8) bezeichnen. Nichtsdestotrotz integriert ihr Zugang weitere soziologische Traditionen, die ihrer feldtheoretischen Perspektive eine andere Stoßrichtung verleihen. Die disziplinären Hintergründe beider Autoren sind dabei zentral. Theoretische Konzepte, die in der Forschung zu sozialen Bewegungen verbreitet sind, wie z.B. Framing-, Ressourcenmobilisierungs- oder Political Opportunity-Ansätze, finden sich ebenso deutlich wieder wie der in der Wirtschaftssoziologie verbreitete soziologische Neoinstitutionalismus. Wenngleich Fligstein, der selbst oft als Neoinstitutionalist verortet wird (Swedberg 2009, S. 73), seine feldtheoretische Perspektive mehrfach von neoinstitutionalistischen Ansätzen (und insbesondere neoinstitutionalistischen Feldansätzen, Powell und DiMaggio 1983) abgrenzt (Fligstein und McAdam 2012a, S. 11 f.; Kluttz und Fligstein 2016), bleibt deren Einfluss z.B. im Fokus auf (industrielle) Normen und Standards oder das 
Konzept der Internal Governance Units, die Kontrollkonzepte institutionalisieren (Fligstein und McAdam 2012a, S. 14), klar erkennbar. Einen weiteren starken Bezugspunkt stellen der Symbolische Interaktionismus und insbesondere die Arbeiten von George H. Mead dar. Berührungspunkte zu Bourdieu zeigen sich hier im Fokus auf Bedeutungen, Symbole und die soziale Konstruktion der Realität. Gleichwohl zeichnet die Orientierung am Symbolischen Interaktionismus (und damit mittelbar auch am amerikanischen Pragmatismus) für eine Reihe von Unterschieden verantwortlich, die Fligsteins Perspektive klar von Bourdieus abgrenzen: den Fokus auf Interaktion, Kooperation und Kommunikation; die relative Aufwertung von Akteuren gegenüber strukturellen Zwängen; den Fokus auf gegenwärtige Situationen und die relative Vernachlässigung der Vergangenheit; sowie das zentrale Interesse an Identität und Gemeinsamkeit anstelle von Distinktion und Abgrenzung. Die Feldtheorie Fligsteins' schließt somit an eine Theorietradition an, die in zentralen Aspekten von der Bourdieu'schen Perspektive abweicht.

Weitere Unterschiede sind im methodologischen Zugang erkennbar. Zwar arbeitet auch Fligstein seit Beginn seiner wissenschaftlichen Karriere explizit historisch (vgl. Fligstein 1981), die Analyse erfolgt jedoch auf einem wesentlich abstrakteren und stärker aggregierten Niveau. Dies lässt sich besonders eindrücklich an einem Untersuchungsgegenstand verdeutlichen, dem sich sowohl Fligstein als auch Bourdieu zugewandt haben: der Genese und Funktionsweise von Dispositionen im Finanzbereich (Bourdieu et al. 1963; Fligstein und Goldstein 2015). Während Bourdieu und Kollegen hier eine Feldanalyse präsentieren, die auf umfangreichen Interviews, Feldbeobachtungen und qualitativer Dokumentenanalyse basiert und damit die alltäglichen Praktiken von Banken und Bankkunden greifbar macht, stützen Fligstein und Goldstein ihre Studie auf standardisierte, stark aggregierte Umfragedaten zu finanzorientierten Einstellungen der US-amerikanischen Bevölkerung, die mittels Regressionsanalyse verschiedenen soziodemografischen Variablen im Sinne eines Hypothesentests gegenübergestellt werden. Der Gebrauch von Regressionsanalysen, der für Fligstein typisch ist, entspricht dem Denken in abhängigen und unabhängigen Variablen. Wohl auch deshalb scheint das Fligstein'sche Instrumentarium die für Bourdieu zentralen „objektiven Relationen“ weniger deutlich in den Fokus zu rücken, was z.B. hinsichtlich des Ressourcenbegriffs deutlich wird. Während Ressourcen für Bourdieu ausschließlich relativ zum Feld gefasst werden können (s.u.), stellt Fligstein stärker auf absolute Faktoren, wie z.B. Profit ab. Der Rückgriff auf abstrakte, quantitative Indikatoren verstärkt zudem eine theoretische Berücksichtigung von Faktoren, die offen zutage treten und dadurch eindeutig messbar sind. Die (im Verhältnis zur Bourdieu'schen Perspektive) Vernachlässigung verborgener Machtressourcen und Machtkämpfe ist somit ein Stück weit dem methodologischen Zugang geschuldet (Medvetz 2013).

\subsection{Untersuchungsinteressen: Schwerpunkte und zentrale Konzepte}

Entlang der verschiedenen Entstehungskontexte wurden bereits eine Reihe konzeptioneller Unterschiede angedeutet. Im Folgenden gilt es, diese nochmals dezidiert herauszuarbeiten. Die Darstellung orientiert sich dabei an sechs zentralen Aspek- 
ten, an denen Differenzen deutlich werden: Akteurshandeln, Historizität, Wandel, Kooperation, Machtressourcen und Kritisches Potenzial.

Akteurshandeln. Ein erster Unterschied zeigt sich am Stellenwert, den beide Theorieansätze der sozialen Struktur gegenüber intentionalem Akteurshandeln zuweisen. Bereits die von Fligstein gewählte Bezeichnung „Strategic Action Fields“ legt den Fokus klar auf strategisch agierende Akteure. Im Anschluss an den amerikanischen Pragmatismus werden strukturalistische Erklärungen abgelehnt und den Akteuren erhebliche Freiheitsgrade zugesprochen (Swartz 2014). Insbesondere betont Fligstein, dass Akteure nicht an unhinterfragbare, informelle Institutionen gebunden sind, sondern die Situationen, in denen sie sich befinden, wie auch die Regeln, nach denen Felder strukturiert sind, aktiv infrage stellen können (Fligstein und McAdam 2012a, S. 17; Kluttz und Fligstein 2016, S. 194f.). Sie vermögen daher, tiefgreifenden Wandel anzustoßen und, indem sie Kooperationspartner finden, radikal neue Ordnungen zu etablieren. Angetrieben werden die Fligstein'schen Akteure dabei nicht ausschließlich durch den Wunsch, die eigene Position zu verbessern, sondern auch von ihrem ,natürlichen“ sozialen Bedürfnis nach Zugehörigkeit, geteilter Identität und einer gemeinsamen Sicht auf die Welt (Fligstein und McAdam 2012a, S. 35 f.). Für Fligstein steht kollektiv motiviertes, jedoch intentional reflektiertes Handeln im Zentrum seiner feldtheoretischen Analyse. Das theoretische Instrumentarium fokussiert auf einen Handlungsmodus, der für ökonomische und politische Felder, in denen Wettbewerb offen ausgetragen wird, typisch scheint. Andere Handlungsmodi - insbesondere solche, die auf vorreflexiven Dispositionen basieren geraten aus dem Blick.

Eine gewisse Ähnlichkeit zur Bourdieu'schen Perspektive besteht darin, dass auch Bourdieu einen starken Strukturalismus, der die Akteure und ihr Handlungspotenzial völlig außer Acht lässt, ablehnt. Ohne Frage schreibt Bourdieu jedoch strukturellen Einflüssen größere Prägekraft zu als Fligstein. Er interessiert sich vor allem für die verborgenen Effekte dieser Strukturen, die hinter dem Rücken der Akteure, jenseits ihrer Reflexionsfähigkeiten, wirken. Das Konzept des Habitus ist für dieses Verständnis zentral. Es umschreibt inkorporierte Denk-, Wahrnehmungs-, Bewertungsund Handlungsschemata, aber - vermittelt über diese - auch komplexere Dispositionen wie Präferenzsysteme, Rationalitätslogiken oder Wirtschaftsgesinnungen. Der Habitus eines Akteurs ist wandelbar, wird jedoch maßgeblich durch dessen soziale Umgebung, d.h. dessen vergangene und aktuelle Feldposition bestimmt. Zwar betont Bourdieu immer wieder, dass sein Habituskonzept Akteure eben nicht zu Marionetten einer objektiven Struktur degradiert (Bourdieu 1998b, S. 198f.), sondern der Habitus stattdessen wie ein Filter zwischen externen Reizen und tatsächlichen Praktiken vermittelt und so die Genese individueller Strategien, d.h. einer ,geregelten Improvisation“ (Bourdieu 1976, S. 179) erlaubt. Nichtsdestotrotz ist wirklich reflexives Handeln, das an einem Kalkül orientiert ist, welches über die eigene Prägung hinausblickt, für Bourdieu eine seltene Ausnahme. Die wechselseitige Bedingtheit von Habitus und Feld lässt wenig Platz für kreative Strategien und Versuche, die Situation grundlegend zu verändern - Strategien also, die für Fligstein im Mittelpunkt des Interesses stehen. Aus Bourdieus Perspektive ist es der Habitus, der bestimmt, welche Strategien für die Akteure überhaupt denkbar sind. Seiner Feldtheorie wurde daher häufig ein mehr oder minder latenter „Determinismus“ vorgeworfen (Sewell 
1992; Kluttz und Fligstein 2016, S. 192; vgl. Lebaron 2003). Die Kritik, die sich an diesem Punkt entzündet, hat nicht zuletzt die Entstehung der économie des conventions (Diaz-Bone 2015) befördert, einer französischen Schule, die hier bewusst mit Bourdieu bricht und die reflexiven Fähigkeiten von Akteuren betont (Boltanski und Thévenot 2007, S. 203). Zwar liegt der Reiz der Bourdieu'schen Perspektive gerade darin, der Illusio ökonomischer Akteure nicht aufzusitzen, d.h. Handeln, das diese als rational-intentional ausgeben, als Ergebnis objektiver Feldpositionen und inkorporierter Dispositionen zu entlarven. Gerade für die Analyse ökonomischer Bereiche scheint es jedoch problematisch, die Erklärung entlang intentionaler Strategien derart auszuklammern (Maurer 2006).

Historizität. In der Bourdieu'schen Semantik ist das dargestellte Habituskonzept aufs Engste mit dem Aspekt der Historizität verbunden. Auch Neil Fligstein arbeitet fast ausschließlich historisch und betrachtet Wandel und Stabilisierung von empirischen Feldern über die Zeit. Allerdings spielt die Historizität, verstanden als das „So-und-nicht-anders-geworden-sein“ (Weber 1904, S. 60) von Feldern, Regeln, Machtressourcen und Dispositionen in seiner feldtheoretischen Perspektive keine starke oder gar konzeptionelle Rolle. Es geht um das überblicksartige Erfassen von Entwicklungen und Umbrüchen und nicht um eine detaillierte sozialwissenschaftliche Genealogie. Im Sinne der pragmatischen Tradition, in der die Fligstein'sche Perspektive steht, liegt der Fokus auf der Situation: Das Handeln und Entscheiden von Akteuren wird stärker aus der gegenwärtigen Interaktion mit Kooperationspartnern und weniger aus der historischen Prägung heraus erklärt (Swartz 2014). Ökonomische Koordination erscheint aus dieser Perspektive situationsgebunden.

Für Bourdieu stellt Historizität hingegen ein fundamentales Prinzip sozialwissenschaftlichen Erklärens dar. Es findet sich nicht nur im Zuschnitt einzelner empirischer Studien, sondern auch im konzeptionellen Instrumentarium seiner Feldtheorie wieder: sowohl im Konzept des Feldes als „Ding gewordener“ als auch des Habitus als „Leib gewordener Geschichte“ (Bourdieu 1985, S. 69). Der Habitus lässt sich weniger durch die aktuelle Situation als vielmehr durch frühere Positionen, d.h. ,die Spur der bisher durchlaufenen Bahn“ (Bourdieu 1998b, S. 198) erklären. Die Vergangenheit ist somit sowohl durch die erworbenen Dispositionen als auch durch die entstandenen Gelegenheitsstrukturen in der Gegenwart präsent. Mehr noch bedingt die Vergangenheit für Bourdieu selbst die Art und Weise, wie Akteure über die Zukunft denken (können) (2000). Für die wirtschaftssoziologische Analyse impliziert dies, dass sich Koordination in ökonomischen Feldern nur verstehen lässt, wenn Geschichte explizit als erklärender Faktor mit einbezogen wird: Ökonomische NormStrategien, Professionen, Best Practices, Organisationsprinzipien und Kennzahlen, aber auch Wirtschaftsgesinnungen oder ökonomische Zukunftserwartungen werden erst durch die Betrachtung ihrer jeweiligen historischen Genese erklärbar.

Wandel. Ein elementarer Vorteil seiner feldtheoretischen Perspektive, so betont Fligstein, liege darin, nicht nur die Stabilität von Feldern, sondern auch deren Wandel und Entstehung theoretisch fassen zu können. In der Tat beinhaltet die Fligstein'sche Feldtheorie ein systematisches Phasenmodell, das beschreibt, unter welchen Bedingungen Wandel oder Reproduktion stattfinden (Fligstein und McAdam 2012a, S. 83 ff.). Der Entstehungsprozess von Feldern wird, ausgehend von McAdams Forschungsschwerpunkt (McAdam 1990, 1999), ähnlich der Genese sozia- 
ler Bewegungen konzeptualisiert, von Fligstein dann aber später auch auf die Genese neuer Märkte übertragen (Fligstein 2010, S. 87 ff.). Emergierende Felder sind demnach von Unsicherheit und starken Dynamiken geprägt. Erst allmählich gelingt es den Akteuren, Koalitionen und Netzwerke zu mobilisieren, um ihr präferiertes Kontrollkonzept durchzusetzen. Hierbei wird der „sozialen Kompetenz“ der Akteure (s.u.) zentrale Bedeutung beigemessen. Zur weiteren Stabilisierung von Feldern tragen dann der Staat mit seiner Fähigkeit, sanktionierbare Institutionen durchzusetzen, sowie neu etablierte Internal Governance Units, die Kontrollkonzepte sichtbar festschreiben, bei. Externe Faktoren, wie z.B. Verschiebungen und Überschneidungen im System der Felder, können die Genese neuer Felder begünstigen. Stabile Felder charakterisieren sich der Fligstein'schen Perspektive folgend durch beständige, geteilte Kontrollkonzepte, die Identität stiften. Das zunehmende Infragestellen herrschender Kontrollkonzepte sowie die schwindende Fähigkeit etablierter Akteure, ihre Position zu reproduzieren, sind demzufolge wichtige Anzeichen für eine voranschreitende Destabilisierung und Krise des Feldes. Die Reorganisation und erneute Stabilisierung des Feldes ähnelt dann den Machtkämpfen und Koalitionsbemühungen in neuen Feldern - und gipfelt in der Etablierung eines neuartigen Kontrollkonzepts (Fligstein 2010, S. 75 ff.). Diese sehr eingängige Beschreibung bietet gewissermaßen eine Theorie des Wandels, die unabhängig vom empirischen Einsatzbereich ,,prêt à l'emploi“ (Medvetz 2013) ein systematisches Erklärungsangebot vorhält. Indem sie die Reduktion von Unsicherheit ins Zentrum ihrer Analyse stellt, ist sie höchst anschlussfähig zur NWS und deren Einbettungsperspektive. Die Fligstein'sche Feldtheorie lenkt den Fokus dabei auf die charakteristische Dynamik ökonomischer Felder: das Entstehen und Verglühen von neuen Trends, Unternehmenstypen oder Märkten.

Im Gegensatz dazu wird dem Ansatz Bourdieus häufig (u.a. von Fligstein und McAdam 2012a, S. 25f.) vorgeworfen, blind für Wandel und Innovation zu sein, da er lediglich Interesse an der Reproduktion einmal etablierter Strukturen zeige. Bei genauerer Lektüre trifft dies nur bedingt zu. Erstens hat Bourdieu in seinen eigenen empirischen Studien immer wieder Genese und Wandel von Feldern in den Blick genommen (z.B. Bourdieu 1998c, 1999) - auch seine bekannteste wirtschaftssoziologische Studie, Der Einzige und sein Eigenheim (1998b), analysiert die Genese des Marktes für Fertighäuser und zeigt nicht zuletzt auf, wie der Staat auf dieses neue Feld signifikanten Einfluss nimmt. Zweitens belegen Bourdieus Interventionen als Intellektueller seine Überzeugung, dass sozialer Wandel möglich ist (Costa 2006). Drittens finden sich entlang des Bourdieu'schen Gesamtwerks immer wieder Hinweise, dass seine theoretischen Konzepte - Feld, Habitus und Kapital auch für die Analyse von Wandel genutzt werden können: ,Was die Struktur des Feldes ausmacht, wie ich sie sehe, [ist] auch das Prinzip seiner Dynamik“ (Bourdieu 1993, S. 191). Davon unberührt liegt ein wesentliches Interesse der kritischen Perspektive Bourdieus sicher darin, die Stabilität sozialer Strukturen aufzuzeigen und zu erklären. Immer wieder zeigt Bourdieu, dass soziale Strukturen sich trotz und gerade wegen der Transformationsversuche der Dominierten hinter dem Rücken der Akteure reproduzieren. Wenngleich die Reduktion von Unsicherheit für Bourdieu eine untergeordnete Rolle spielt, eröffnet dieser Zugang neue Perspektiven für die Wirtschaftssoziologie: Er lenkt den Blick hinter die Fassade sich scheinbar un- 
ablässig wandelnder Märkte, Unternehmen oder Volkswirtschaften und deckt die Reproduktion ökonomischer Ungleichheiten, die sich unterhalb dieser dynamischen Oberflächen vollzieht, auf.

Kooperation. Am deutlichsten unterscheiden sich die beiden feldtheoretischen Ansätze im Hinblick auf die Bedeutung, die sie Kooperation beimessen. Geprägt durch die Tradition des symbolischen Interaktionismus zeigt Fligstein erhebliches Interesse an der Rolle von Kooperation, Interaktion und Kommunikation. Neben objektiven, latenten Relationen räumt die Fligstein'sche Feldtheorie damit, ähnlich wie das in der NWS verbreitete Einbettungskonzept, auch persönlichen Interaktionsbeziehungen Platz ein. Dies findet nicht zuletzt im Konzept des „social skill“, seinen Ausdruck (Fligstein 1997; Fligstein und McAdam 2012a, S. 45 ff.). Unter sozialer Kompetenz wird hier die Fähigkeit verstanden, sich in andere hineinzuversetzen, sie anzusprechen und zu mobilisieren, um so möglichst breite Koalitionen und tragfähige Kompromisse zu etablieren. Soziale Kompetenz ermöglicht kooperatives Handeln und wird aus dem menschlichen Grundbedürfnis nach Zugehörigkeit, gemeinsamer Identität und gemeinsamer Weltsicht abgeleitet. Mit dieser Konzeption von menschlichen Akteuren als homines sociologici grenzt sich Fligstein klar von ökonomischen Rational-Choice-Theorien ab. Die Fähigkeit kollektiv zu handeln und Koalitionen zu schmieden ist, wie oben gezeigt, der zentrale Faktor, der sowohl die Genese neuer Felder, als auch Transformation und Krise existierender Felder erklärt. Koordination kann jedoch auch zur Stabilität und Resilienz von Feldern beitragen. Felder können entweder durch Dominanz und Unterdrückung stabilisiert, oder durch Zusammenhalt, Kooperation und friedlichen Konsens unter den Feldakteuren gefestigt werden (Kluttz und Fligstein 2016, S. 199). Kooperation schließt aus dieser Sicht Machtkämpfe in keiner Weise aus. Die Feldkämpfe werden jedoch durch zwei entgegengesetzte menschliche Motive angetrieben: sich einerseits abzugrenzen und andere zu unterwerfen, aber andererseits ebenso den Wunsch, dazuzugehören und mit anderen zu kooperieren. Für die NWS erlaubt diese feldtheoretische Perspektive einen Fokus auf ihr Kernanliegen der ökonomischen Koordination. So müssen Joint Ventures oder Branchenverbände nicht ausschließlich aus der Sicht eines KostenNutzen-Kalküls analysiert werden: Sowohl Distinktion als auch Sozialität können als Erklärungsfaktoren genutzt werden.

Bourdieu hat jede interaktionistische Sichtweise unverhohlen abgelehnt (z.B. 1998b, S. 181). Die Bourdieu'sche Feldtheorie stellt daher sehr deutlich Kampf, Dominanz und Opposition ins Zentrum der Analyse (und bedient sich hierzu z.B. der Korrespondenzanalyse, die auf der Sichtbarmachung von Oppositionen beruht, s.o.). Kooperation und den damit implizierten Interaktionsbeziehungen wird kaum Beachtung geschenkt, um den Fokus auf den „Strukturzwang des Feldes“ nicht zu vernachlässigen. Allenfalls spielt Kooperation als Machtressource im Sinne von sozialem Kapital eine Rolle (Bourdieu 1983, S. $191 \mathrm{ff}$.). Der Umfang an sozialem Kapital, das ein Akteur zur Verfügung hat, setzt sich zusammen aus der Größe des Netzwerks an Beziehungen, die er mobilisieren kann, sowie dem Umfang an allen innerhalb des Netzwerks verfügbaren Kapitalien (z.B. ökonomischer oder symbolischer Natur). Wenngleich der Bourdieu'sche Begriff des sozialen Kapitals häufig herausgegriffen und interaktionistisch gewendet wurde (Eloire 2014), erhält das Konzept erst seine Bedeutung, wenn es in Verbindung mit Bourdieus umfassendem, 
pluralem Kapitalkonzept gedacht wird. Soziales Kapital kann in andere Kapitalsorten transformiert werden, d.h. es kann z.B. zur Akkumulation von ökonomischem oder symbolischem Kapital beitragen. Zudem prägt es den Habitus seines Inhabers, was es diesem wiederum erlaubt, gewinnträchtige soziale Beziehungen aufzubauen - oder eben nicht. Dabei ist es für Bourdieu, anders als für Fligstein, unwichtig, ob Akteure Netzwerke aus berechnendem Kalkül oder aus affektiven Motiven bzw. einer genuinen Sozialität heraus etablieren - von Relevanz ist für die Bourdieu'sche Feldtheorie einzig der Umstand, dass sie als Ressource wirken und so zur Reproduktion von Ungleichheiten im Feld beitragen.

Machtressourcen. Die Unterscheidung und Analyse von Machtpositionen - d.h. von „objektiven Relationen“ - ist ein wichtiges feldtheoretisches Charakteristikum. Dennoch unterscheiden sich der Fligstein'sche und der Bourdieu'sche Ansatz in ihrer Konzeption von Macht erheblich. Fligstein betont, dass Macht und Konflikt sowie Kämpfe zwischen Herausforderern und Etablierten im Zentrum seiner feldtheoretischen Analyse stehen. Die Ursachen von Macht werden jedoch nicht dezidiert besprochen, stattdessen wird unspezifisch auf ,unterschiedliche Ressourcenausstattung" (Fligstein und McAdam 2012a, S. 10) verwiesen. Lediglich auf die Rolle sozialer Kompetenzen bei der Durchsetzung eigener Kontrollkonzepte wird detaillierter eingegangen. Macht erscheint als offen zutage tretendes Phänomen, das einer einfachen sozialwissenschaftlichen Messung zugänglich ist und eine problemlose Unterteilung in Etablierte und weniger Etablierte erlaubt.

Im Gegensatz hierzu stellt Pierre Bourdieu die Frage, woher Macht kommt und auf welch unterschiedliche Art sie akkumuliert und reproduziert werden kann, in den Mittelpunkt seiner Feldtheorie. Am deutlichsten wird dies am Konzept des Kapitals, das ein ausdifferenziertes, plurales und radikal relationales Verständnis von Machtressourcen offenbart. Kapital umreißt Bourdieu, der physikalischen Metapher treu bleibend, als „Energie der sozialen Physik“ (Bourdieu 1976, S. 357), die in unterschiedlichen Formen zutage tritt. Er bietet als erste Heuristik eine Unterscheidung zwischen ökonomischen, kulturellen, sozialen und symbolischen Kapitalien an. Darüber hinaus betont er, dass verschiedenste Fähigkeiten, Eigenschaften oder Besitztümer zum Kapital avancieren können. Was Wert hat und als „Trumpf“ eingesetzt werden kann, wird im jeweiligen Feld - d.h. relativ dazu - definiert und umkämpft (Bourdieu und Wacquant 1996, S. 128). Während z.B. ein Doktortitel im Feld der Management-Beratung als kulturelles Kapital gelten mag, stellt derselbe Titel im Feld des Punk-Rock keine Machtressource dar. Da jedes Kapital für Bourdieu auf akkumulierter Arbeit beruht, sind die verschiedenen Kapitalien prinzipiell transformierbar. So lässt sich genannter Doktortitel beim Ausstieg aus dem wissenschaftlichen Feld etwa über ein erhöhtes Einstiegsgehalt in ökonomisches Kapital umwandeln. Inwiefern derartige Transformationen möglich sind und eine zusätzliche Akkumulation von Kapital erlauben, hängt von den jeweils gültigen Wechselkursen im Feld ab. Diese versteht Bourdieu, ebenso wie die Kapitalien selbst, als umkämpfte Machtinstrumente. Das plurale Verständnis von Machtressourcen, das der Bourdieu'schen Feldtheorie zugrunde liegt, ermöglicht es somit einerseits, dezidiert zu betrachten, welche Formen von Macht eine Rolle spielen und wie sich der Besitz verschiedener Kapitalien ggf. wechselseitig verstärkt. Darüber hinaus werden, insbesondere über den Begriff des symbolischen Kapitals, auch verborgene Machtkämpfe 
sichtbar. So können Akteure auch darum wetteifern, wer als am wenigsten profitorientiert oder uneigennützig gilt. Auch diese scheinbare „Interesselosigkeit“ kann als wichtige Ressource fungieren, die eine Art von Macht verleiht, die eben nicht offensichtlich ist. Der wirtschaftssoziologischen Analyse wird so ein Instrumentarium eröffnet, das neben den offenen, bewussten Wettkämpfen im ökonomischen Bereich auch verborgene, symbolische Machtkämpfe aufdeckt, die nicht minder zur Reproduktion von ökonomischen Ungleichheiten und damit zur scheinbar mühelosen Koordination in ökonomischen Feldern beitragen (Suckert 2017).

Kritisches Potenzial. Das Untersuchungsinteresse eines theoretischen Ansatzes beschränkt sich nicht nur auf die Schwerpunkte, die durch die Anordnung einzelner Konzepte in den Blick geraten. Zentral ist auch das grundlegende Anliegen, das eine soziologische Theorie verfolgt: Geht es „nur“ darum, einen Erklärungsbeitrag zur empirisch beobachtbaren sozialen Welt zu leisten, oder soll die Theorie auch die Instrumente liefern, um das, was ist, infrage zu stellen und gesellschaftskritische Anliegen zu formulieren? Die Feldtheorien Bourdieus und Fligsteins unterscheiden sich auch in diesem Punkt elementar.

Fligstein geht es mit seiner Feldtheorie in erster Linie darum, soziale Ordnung und sozialen Wandel auf der Meso-Ebene, also z.B. bezogen auf Unternehmen, Märkte oder Sektoren zu erklären. Wie gelingt Stabilität und unter welchen Umständen kann es zu Umbrüchen kommen? Fligstein bietet einen theoretischen Werkzeugkasten, der es erlaubt, in Bezug auf diese Fragen Hypothesen aufzustellen und diese empirisch zu testen. Kritisch ist dieser Zugang nur gegenüber den in den Wirtschaftswissenschaften populären Rational-Choice-Ansätzen. Deren Unzulänglichkeit soll durch bessere, da feldtheoretische Erklärungsangebote nachgewiesen werden. Die Fligstein'sche Theorie nimmt hier, ganz im Sinne der NWS, die Position des Herausforderers ein. In der stetigen Betonung staatlicher Akteure als Garanten für stabile Felder lässt sich allenfalls ein sozialdemokratischer Appell zur Beibehaltung eines starken Staates erkennen - auch dies eine Kritik an der Lehrmeinung neoklassischer Ökonomen.

Den Anspruch, selbst Gesellschaftskritik mit seiner Feldtheorie zu betreiben, erhebt Fligstein nicht. Jedoch sind die Akteure, die er konzipiert, mit einer starken Kritikfähigkeit ausgerüstet: Sie können sich eine andere Welt jenseits dessen, was ist, vorstellen. Die Fligstein'sche Feldtheorie bietet zwar nicht die Instrumente, die eine alternative Sicht auf die Welt befördern, sie gibt aber Aufschluss darüber, unter welchen Bedingungen sich eine solche Sicht durchsetzen kann und sozialer Wandel möglich wird. In diesem Sinne kann sie, im Anschluss an die Selbstbeschreibung von Luc Boltanski und der économie des conventions (Boltanski 1990), als ,Soziologie der Kritik“ aufgefasst werden. Eine kritische Soziologie im engeren Sinne ist sie jedoch nicht.

Im Gegensatz hierzu ist die Feldtheorie Bourdieus durch und durch von dem Anspruch geprägt, kritische Soziologie zu betreiben - ungeachtet der Tatsache, dass sich Bourdieu von der kritischen Theorie der Frankfurter Schule wiederholt abgegrenzt hat (Bauer et al. 2014). Seit seinen frühen Algerienstudien geht es Bourdieu im Kern darum, verborgene Macht- und Herrschaftsverhältnisse aufzudecken und anzuprangern. Diese kritische Haltung ist jedem seiner theoretischen Konzepte von Anfang an eingeschrieben: Das Konzept des Kapitals dient dazu, verborge- 
ne Ressourcen aufzudecken, ungleiche Ausgangsbedingungen zu beschreiben und aufzuzeigen, wie Privilegien aus einem Bereich Vorteile in einem anderen Bereich ermöglichen. Das Konzept des Habitus, der in weiten Teilen unbewusst, aber inkorporiert ist und dem Akteure daher nicht entkommen können, deckt auf, warum Akteure blind an Praktiken festhalten, die objektiv unvorteilhaft für sie sind und sie schlechter stellen. Die symbolische Herrschaft, d.h. eine Unterwerfung, die sich die Unterworfenen zu Komplizen macht, wird so fassbar. Das Konzept der Feldstruktur zeigt ungleiche Positionen auf, die den Möglichkeitsraum der Akteure beschränken und sich, zumeist hinter dem Rücken der Akteure, reproduzieren. Indem Bourdieu der Soziologie eine „klinische, ja therapeutische Funktion“ (1992, S. 223) beimisst und ihre „befreiende Kraft“" (1993, S. 44) beschwört, zeigt sich, wie sehr er auch seine Feldtheorie als emanzipatorisches Projekt begreift, das dazu dient, die Menschheit - ganz im Kant'schen Sinne - aus der „selbstverschuldeten Unmündigkeit“ zu führen. Die Bourdieu'sche Feldtheorie bietet damit, wie die Fligstein'sche auch, ein Instrumentarium, um ökonomische Koordination auf der Meso-Ebene nachzuvollziehen und zu erklären - darüber hinaus aber auch eines, um die Formen dieser Koordination kritisch zu hinterfragen.

Insgesamt zeigt sich, dass die Fligstein'sche und die Bourdieu'sche Feldtheorie trotz einiger gemeinsamer Grundlagen verschiedenen sozialwissenschaftlichen Traditionen entspringen, unterschiedliche Schwerpunkte setzen und verschiedene erklärungstheoretische Ansprüche erheben. Mit Blick auf die wirtschaftssoziologische Analyse weisen sie daher unterschiedliche Potenziale und Schwachstellen auf: Während Fligsteins Theorie, die bereits dezidiert als wirtschaftssoziologische Theorie entworfen wurde, viele Themen der NWS aufgreift und problemlos anschlussfähig scheint, bietet gerade die andersartige Bourdieu'sche Perspektive Inspiration, um die wirtschaftssoziologische Agenda über allgemeine feldtheoretische Elemente hinaus zu erweitern.

\section{Der Mehrwert einer wechselseitig informierten Lektüre für eine feldtheoretische Perspektive auf die Wirtschaft}

Ausgehend vom verschiedenartigen Zuschnitt beider Ansätze, scheint eine Integration im engen Wortsinn wenig tragfähig. Wie gezeigt, greifen beide Theorien trotz grundlegender Gemeinsamkeiten auf unterschiedliche und in ihren Annahmen teils widersprüchliche soziologische Traditionen zurück, die ihren Ursprung jenseits und diesseits des Atlantiks haben. Eine umfassende Verbindung wäre unweigerlich von einem gewissen Eklektizismus gezeichnet. Stattdessen soll in diesem Abschnitt eine wechselseitig informierte Lektüre angeregt werden. Was gewinnt man, wenn man Bourdieus feldtheoretische Arbeiten aus einer Fligstein'schen Perspektive liest? Welche Aspekte der Fligstein'schen Theorie erscheinen aus einem Bourdieu'schen Blickwinkel ergänzungswürdig? Ziel ist dabei nicht die Genese einer ,neuen“ Feldtheorie, sondern die Erweiterung des jeweiligen konzeptionellen Horizontes. Die wechselseitige Befruchtung soll Anhaltspunkte für ein wirtschaftssoziologisches, aber feldtheoretisches Forschungsprogramm (vgl. Bernhard und Schmidt-Wellenburg 2012) geben, das sowohl auf der Fligstein'schen, als auch auf der umfassende- 
ren Bourdieu'schen Perspektive und den jeweiligen Annahmen fußen kann. Um deutlich zu machen, dass der Mehrwert dabei gerade den divergierenden Blickwinkeln beider Ansätze entspringt, stehen - analog zum vorherigen Abschnitt - wiederum die Aspekte Akteurshandeln, Historizität, Wandel, Kooperation, Machtressourcen und das resultierende kritische Potenzial im Mittelpunkt der Betrachtung.

Akteurshandeln. Die wirtschaftssoziologische Anwendung der Bourdieu'schen Feldtheorie leidet darunter, dass reflexives, intentionales Handeln von Bourdieu als Ausnahme konzipiert wird. Konfrontiert mit einem Gegenstandsbereich, in dem ganze Hundertschaften auf die Analyse von „Marktpositionen“ und „Marktstrategien“ angesetzt werden, Managementtools zur Reflexion der eigenen Schwächen und Stärken ermahnen, Kreativitätstechniken eingesetzt werden, um ,outside the box“ zu denken, und die bewusste Wahrnehmung von Eigeninteresse als Tugend gilt, erscheint es wenig überzeugend, intentionales und reflexives Handeln per se als habituellen Reflex abzutun. Die Fligstein'sche Perspektive kann hier Impulse für eine weniger rigide Lesart des Habituskonzepts geben, die die von Bourdieu vorgebrachten Weiterentwicklungen ernst nimmt. ${ }^{9}$ So betont Bourdieu einerseits die Besonderheiten des ökonomischen Habitus, dessen ,bedingte und begrenzte Spontanität" (Bourdieu 1998b, S. 216) zu einem höheren Freiheitsgrad in ökonomischen Feldern führt. Er deutet damit implizit an, dass bewusst strategisches Verhalten in ökonomischen Feldern, wenn auch nur in sehr begrenztem Maße, möglich ist (Müller 2014, S. 248). Zum anderen findet sich in Bourdieus Werk wiederholt der Hinweis, dass habituell geprägtes Handeln nur ein Handlungsmodus unter anderen ist (z.B. 1976, S. 207, 1992, S. 99). Der Kern des Problems liegt damit nicht in einem Determinismus per se, sondern darin, dass Bourdieu keine Aussage dazu macht, in welchem Verhältnis der Habitus zu anderen, reflexiven Handlungsprinzipien steht. Es bleibt unklar, wann und unter welchen Bedingungen welches Prinzip zum Tragen kommt und wie die verschiedenen Prinzipien interagieren können (Maurer 2006, S. $136 \mathrm{ff}.) .^{10}$

Für die Fligstein'sche Konzeption von Akteuren hält die Bourdieu'sche Perspektive umgekehrt die Einsicht bereit, dass die beschriebenen ,freien“ ökonomischen Akteure und deren Reflexionsfähigkeit eben keine anthropologischen Konstanten sind, sondern das Produkt einer spezifischen Sozialisation und entsprechender Feldkräfte. Umbrüche, wie z.B. die Gründung neuer Unternehmen oder Märkte unterliegen damit einer weiteren, von Fligstein wenig beachteten Vorbedingung: der Genese eines modernen, spezifisch ökonomischen Habitus, der explizit auf Wandel und Fortschritt ausgerichtet ist. Der Mehrwert für eine wirtschaftssoziologische Betrachtung könnte somit in einer Perspektive liegen, die einerseits den besonderen Handlungsmodus ökonomischer Felder ernst nimmt, diesen aber andererseits als voraussetzungsvoll versteht. Die Bedingungen seiner Entstehung (z.B. die Performativität ökonomi-

\footnotetext{
9 Schmitz (2016, S. 109 ff.) regt dazu an, selbst Rational-Choice-Theorien als Sonderfall der Feld-HabitusTheorie zu verstehen.

${ }^{10}$ Hilfreich erscheint hier die Perspektive der économie des convenctions, die sich u.a. mit dem Übergang zwischen verschiedenen Handlungsregimen bzw. verschiedenen Formen des Engagiertseins befasst (Boltanski 2012; Thévenot 2007) und damit den Übergang von habituellem zu reflexivem Handeln greifbar macht.
} 
scher Theorien) könnten ebenso wirtschaftssoziologisch untersucht werden wie die Wechselwirkungen verschiedener reflexiver und vorreflexiver Modi des Handelns.

Historizität. Die Betonung von Geschichtlichkeit ist eine besondere Stärke der Bourdieu'schen Feldtheorie, von der auch die Fligstein'sche Perspektive profitieren könnte. Feldakteure, Regeln und Strategien würden dann zwar immer noch durch die aktuelle Situation, aber eben auch aus ihrem „Gewordensein“ heraus erklärt. Diese Perspektive scheint insbesondere für das Verständnis der Internal Governance Units, denen Fligstein große Bedeutung beimisst, fruchtbar. So lässt sich die Funktionsweise und Autorität von z.B. Zertifizierungsstellen, Industrieverbänden oder Standardisierungsorganisationen oft nur aus deren ursprünglicher Genese und Umwidmung heraus erklären. Ebenso erscheint es sinnvoll, das Verhältnis zwischen Feldern, das Fligstein umfassend systematisiert, im Hinblick auf Geschichtlichkeit zu analysieren: Gesamtgesellschaftliche Prozesse, die für ökonomische Bereiche eine wichtige Rolle spielen, wie z.B. Liberalisierung, Quantifizierung oder Globalisierung (Bernhard und Schmidt-Wellenburg 2014), können so als Verschiebungen zwischen Feldern gefasst werden.

Wandel. Bourdieus Verengung auf die Reproduktion sozialer Ordnung ist bei Weitem nicht so absolut, wie seine Kritiker ihm vorwerfen (s. Abschnitt 4.2). Fligsteins gut systematisierte Überlegungen zum Thema Wandel können jedoch Anlass geben, das Bourdieu'sche Werk mit einem Fokus auf Dynamik zu rekapitulieren und die enthaltene bruchstückhafte Darstellung zusammenzuführen. ${ }^{11}$ So lassen sich auch mit Bourdieu drei Typen von Wandel unterscheiden (Suckert 2015, S. 94 ff.): Erstens oberflächlicher Wandel bzw. „ständige Teilrevolutionen“, die „die Grundlagen des Spiels selbst“" (Bourdieu 1993, S. 110) nicht verändern. Zweitens Krisen des Feldes, die zu fundamentalem Wandel führen, wenn es gelingt, externe Einflüsse in interne Machtkämpfe zu übersetzen (Bourdieu 1999, S. 400f.). Sowie drittens die Genese neuer Felder, die durch die Abspaltung von bestehenden Feldern entstehen (1999, S. 100f.). Ähnlich wie Fligstein seinen Internal Governance Units misst auch Bourdieu der Entstehung von Konsekrationsinstanzen erhebliche Bedeutung bei (Bourdieu 1969, S. 90).

Das von Bourdieu vertretene Konzept von Wandel kann die Fligstein'sche Systematik durchaus erweitern: Mit dem Habitus wird ein weiterer Faktor ins Spiel gebracht, der nicht nur Stabilität, sondern auch Umbrüche begünstigen kann. Die Nicht-Passung eines Habitus gibt Anlass zu allerlei „Verrücktheiten“ und damit zu Innovationen im Feld. Fruchtbar erscheint hier insbesondere der Verweis auf die Bourdieu'schen „Doppelwesen“ (Bourdieu und Wacquant 1996, S. 185 f.), die aufgrund ihrer Sozialisation mit einem doppelten Habitus (Bourdieu 1998a, S. 187 f.) ausgestattet sind und daher besonders gut und von innen heraus die Eigenheiten des Feldes infrage stellen und Wandel vorantreiben können. Das Konzept der Doppelwesen zeigt sich zudem anschlussfähig an in der Organisationsforschung verbreitete Ansätze zu „,hybrid actors“ oder ,institutional logics“, die auch Fligstein als wertvolle Ergänzung einer feldtheoretischen Perspektive ansieht (Kluttz und Fligstein 2016, S. 202).

${ }^{11}$ Boyer (2003) interpretiert die Bourdieu'schen Anmerkungen aus der Sicht der Regulationstheorie und kommt zu einem sehr ähnlichen Schluss. 
Kooperation. Ohne Frage stellen kooperative Beziehungen die größte Leerstelle der Bourdieu'schen Feldtheorie dar, insbesondere wenn es um die Analyse ökonomischer Koordination geht. Das Konzept der sozialen Kompetenz stellt daher eine sinnvolle Ergänzung dar. Es könnte als eine entscheidende Komponente des sozialen Kapitals aufgefasst werden, d.h. jener Kapitalsorte, der Bourdieu in seinem Werk am wenigsten Beachtung schenkt (Eloire 2014). Während Bourdieu vereinzelt die Existenz einer derartigen „,besonderen Kompetenz“ andeutet (Bourdieu 1983, S. 193), findet sich bei Fligstein eine systematische und stringente Ausarbeitung, die an der menschlichen Empathiefähigkeit ansetzt.

Andererseits kann die Bourdieu'sche Perspektive helfen, die für einen Soziologen recht bemerkenswerte Vermutung, dass soziale Kompetenz in der Bevölkerung normal und damit zufällig verteilt sei (Fligstein und McAdam 2012a, S. 17), kritisch zu überdenken. Der Rückbezug auf das Habituskonzept impliziert, dass derartige Fähigkeiten durch eine spezifische soziale Prägung erworben werden müssen. Bourdieu geht daher auch, plausibel, davon aus, dass ein gewisses ,Beziehungstalent [...] zwischen den sozialen Klassen - und, bei identischer Klassenzugehörigkeit, auch zwischen Individuen unterschiedlicher sozialer Herkunft - sehr ungleich verteilt ist “ (1983, S. 193 FN 17). Hier zeigen sich auch Anschlussmöglichkeiten an die Analyse des modernen Netzwerk-Kapitalismus, in dem jene bessergestellt werden, die über soziale Kompetenz verfügen (Boltanski und Chiapello 2006).

Machtressourcen. Die von Bourdieu vorgelegte, differenzierte und systematische Ausarbeitung verschiedener Machtressourcen lässt sich als Ergänzung des Fligstein'schen Instrumentariums nutzen, ohne dessen Ausrichtung grundlegend zu verändern. Es kann als Heuristik herangezogen werden, um potenzielle Machtressourcen für die empirische Analyse zu erschließen. Auch in ökonomischen Feldern, in denen das Ringen um Vorteile offen zutage tritt, kann die Perspektive so für nicht offensichtliche, d.h. kulturelle, soziale oder symbolische Ressourcen geöffnet werden. Ebenso lassen sich verborgene Machtkämpfe auch dort aufdecken, wo ökonomische Koordination scheinbar friedlich und geräuschlos stattfindet.

Kritisches Potenzial. Der NWS, zu der auch Fligstein zu zählen ist, wird wiederholt vorgeworfen, ökonomische Grundannahmen zu wenig kritisch zu hinterfragen (Sparsam 2015). Die Bourdieu'sche Feldtheorie kann hier als Gegengewicht fungieren und eine kritisch(er)e Wendung feldtheoretischer Konzepte anmahnen. Für den Fligstein'schen Ansatz erscheint z.B. fraglich, ob soziale Kompetenz als per se positiv und die friedliche Koexistenz fördernd gedacht werden muss. Zwar verweist Fligstein vereinzelt darauf, dass soziale Kompetenz auch für negative Zwecke wie Kreuzzüge oder die Propaganda Adolf Hitlers missbraucht wurde (Kluttz und Fligstein 2016). In seinem Kernbereich, der wirtschaftssoziologischen Analyse, erscheint soziale Kompetenz aber weitgehend als positive, stabilisierende Kraft, die Unsicherheit generell reduziert und Koordination ermöglicht. Auch in ökonomischen Feldern sollte die subtile Macht sozialer Kompetenz jedoch kritisch betrachtet werden, z.B. im Hinblick auf Manipulation, politische Einflussnahme, Unterdrückung von Whistleblowern oder Kartellbildung - wie jüngst im Fall der Automobilbranche zu besichtigen. Die Wirtschaftssoziologie könnte insgesamt von einer Perspektive profitieren, der es nicht nur darum geht, ökonomische Koordination zu erklären, sondern diese auch gesellschaftskritisch zu hinterfragen. 


\section{Fazit}

Der vorliegende Artikel hatte zum Ziel, die beiden Feldtheorien Bourdieus und Fligsteins durch eine systematische Gegenüberstellung ins Gespräch zu bringen. Dabei wurden zunächst eine Reihe von Gemeinsamkeiten herausgearbeitet, die die Basis einer feldtheoretischen und im Kern relationalen Perspektive auf die Wirtschaft bilden. Analog zur NWS grenzt sich diese vom wirtschaftswissenschaftlichen Mainstream und dessen grundlegenden Rational-Choice-Annahmen ab. Die Agenda der NWS wird jedoch in drei zentralen Punkten erweitert:

1) Ein feldtheoretischer Zugang bestärkt den beobachtbaren ,cognitive turn“ der Wirtschaftssoziologie, betont jedoch die umkämpfte Natur kognitiver Phänomene und deren Funktion als Machtinstrumente.

2) Ein feldtheoretischer Zugang ergänzt den wirtschaftssoziologischen Fokus auf Koordination um ein Interesse an Konflikten und Machtbeziehungen. Ungleichheit, ein wichtiges soziologisches, aber von der NWS bislang kaum beachtetes Thema, rückt so ins Zentrum der Analyse.

3) Ein feldtheoretischer Zugang erlaubt der NWS eine ganzheitlichere Perspektive, die die Interaktion und wechselseitige Interdependenz verschiedener wirtschaftlicher und nicht-wirtschaftlicher Bereiche und Ebenen in den Blick nimmt. Dadurch ergeben sich nicht zuletzt Anknüpfungspunkte für die Politische Ökonomie.

Darüber hinaus hat die vorliegende Gegenüberstellung die vielfältigen Unterschiede zwischen beiden Ansätzen deutlich gemacht. Sie bieten für die wirtschaftssoziologische Analyse je verschiedene Potenziale und Desiderate:

Die Fligstein'sche Perspektive kann der NWS vor allem mit ihrem Interesse an Dynamik und Wandel eine neue Stoßrichtung geben. Darüber hinaus bleibt sie mit ihrem Fokus auf reflexivem, intentionalem Handeln sowie auf situativer Kooperation und Interaktionsbeziehungen, die zur Reduktion von Unsicherheit eingesetzt werden, sehr eng am Verständnis der NWS von „eingebettetem“ ökonomischem Handeln. Fligstein bietet der NWS eine überaus anschlussfähige und gut operationalisierbare Systematik, jedoch nur eingeschränkt Impulse für eine Erweiterung des wirtschaftssoziologischen Horizontes über die gängigen Einbettungsansätze hinaus.

Die Bourdieu'sche Feldtheorie zeigt sich dem gegenüber weit weniger anschlussfähig an die Untersuchungsinteressen der NWS und das Konzept der Einbettung. Mit ihrer kritischen Perspektive, die vorreflexives Handeln, verborgene Machtressourcen und Machtkämpfe sowie das „Gewordensein“ ökonomischer Koordination ins Zentrum stellt, kann sie aber gerade deswegen inspirierend für eine Neuausrichtung der wirtschaftssoziologischen Agenda sein. Der gesellschaftskritische Anspruch, der den klassischen wirtschaftssoziologischen Arbeiten noch innewohnte, könnte somit durch die NWS erneut aufgegriffen werden.

Für die Weiterentwicklung eines solchen feldtheoretischen wirtschaftssoziologischen Forschungsprogramms hat die vorliegende Analyse eine wechselseitig informierte Lektüre beider Ansätze vorgeschlagen. Die dargestellten Überlegungen zeigen, dass beide sich gegenseitig ergänzen und inspirieren können - und so das Instrumentarium für eine fruchtbare feldanalytische und relationale Analyse wirtschaftlicher Phänomene bereitstellen. 
Funding Open access funding provided by Max Planck Society.

Open Access Dieser Artikel wird unter der Creative Commons Namensnennung 4.0 International Lizenz (http://creativecommons.org/licenses/by/4.0/deed.de) veröffentlicht, welche die Nutzung, Vervielfältigung, Bearbeitung, Verbreitung und Wiedergabe in jeglichem Medium und Format erlaubt, sofern Sie den/die ursprünglichen Autor(en) und die Quelle ordnungsgemäß nennen, einen Link zur Creative Commons Lizenz beifügen und angeben, ob Änderungen vorgenommen wurden.

\section{Literatur}

Aspers, P. (2007). Wissen und Bewertung auf Märkten. Berliner Journal für Soziologie, 17, 431-449.

Bandelj, N., \& Purg, D. (2006). Networks as resources, organizational logic, and change mechanism: the case of private business schools in post-socialism. Sociological Forum, 21, 587-622.

Bauer, U., Bittlingmayer, U. H., Keller, C., \& Schultheis, F. (Hrsg.). (2014). Bourdieu und die Frankfurter Schule. Bielefeld: Transcript.

Beckert, J. (2007). Die soziale Ordnung von Märkten. MPIfG Discussion Paper. http://www.mpifg.de/pu/ mpifg_dp/dp07-6.pdf.

Beckert, J. (2010). How do fields change? The interrelations of institutions, networks, and cognition in the dynamics of markets. Organization Studies, 31, 605-627.

Beckert, J., \& Rössel, J. (2004). Kunst und Preise. Kölner Zeitschrift für Soziologie und Sozialpsychologie, $56,32-50$.

Benzécri, J.-P. (1982). L'analyse des données. Paris: Dunod.

Bernhard, S. (2010). Netzwerkanalyse und Feldtheorie. Grundriss einer Integration im Rahmen von Bourdieus Sozialtheorie. In C. Stegbauer (Hrsg.), Netzwerkanalyse und Netzwerktheorie (Bd. 1). Wiesbaden: VS Verlag für Sozialwissenschaften.

Bernhard, S., \& Schmidt-Wellenburg, C. (Hrsg.). (2012). Feldanalyse als Forschungsprogramm. Wiesbaden: Springer VS.

Bernhard, S., \& Schmidt-Wellenburg, C. (2014). Editorial: Politische Soziologie transnationaler Felder. Berliner Journal für Soziologie, 24, 137-140.

Blasius, J., \& Schmitz, A. (2013). Sozialraum- und Habituskonstruktion. Die Korrespondenzanalyse in Pierre Bourdieus Forschungsprogramm. In A. Lenger, C. Schneickert, \& F. Schumacher (Hrsg.), Pierre Bourdieus Konzeption des Habitus (S. 201-218). Wiesbaden: Springer VS.

Boltanski, L. (1990). Sociologie critique et sociologie de la critique. Politix, 10, 124-134.

Boltanski, L. (2012). Love and justice as Competences. Cambridge: Polity.

Boltanski, L., \& Chiapello, É. (2006). Der neue Geist des Kapitalismus. Konstanz: UVK.

Boltanski, L., \& Thévenot, L. (2007). Über die Rechtfertigung: Eine Soziologie der kritischen Urteilskraft. Hamburg: Hamburger Edition.

Bourdieu, P. (1969). Intellectual field and creative project. Social Science Information, 8(2), 89-119.

Bourdieu, P. (1976). Entwurf einer Theorie der Praxis: auf der ethnologischen Grundlage der kabylischen Gesellschaft. Frankfurt a. M.: Suhrkamp.

Bourdieu, P. (1983). Ökonomisches Kapital, kulturelles Kapital, soziales Kapital. In R. Kreckel (Hrsg.), Soziale Ungleichheiten (S. 183-198). Soziale Welt Sonderband 2. Göttingen: Schwartz.

Bourdieu, P. (1985). Sozialer Raum und „Klassen“. Leçon sur la leçon: Zwei Vorlesungen. Frankfurt a. M.: Suhrkamp.

Bourdieu, P. (1987). Sozialer Sinn. Kritik der theoretischen Vernunft. Frankfurt a. M.: Suhrkamp.

Bourdieu, P. (1990). Droit et passe-droit. Actes de la recherche en sciences sociales, 81/82, 86-96.

Bourdieu, P. (1992). Rede und Antwort. Frankfurt a. M.: Suhrkamp.

Bourdieu, P. (1993). Soziologische Fragen. Frankfurt a. M.: Suhrkamp.

Bourdieu, P. (1994). L'emprise du journalisme. Actes de la recherche en sciences sociales, 101, 3-9.

Bourdieu, P. (1998a). Praktische Vernunft: Zur Theorie des Handelns. Frankfurt a. M.: Suhrkamp.

Bourdieu, P. (1998b). Der Einzige und sein Eigenheim. Hamburg: VSA.

Bourdieu, P. (1998c). Über das Fernsehen. Frankfurt a. M.: Suhrkamp.

Bourdieu, P. (1999). Die Regeln der Kunst: Genese und Struktur des literarischen Feldes. Frankfurt a. M.: Suhrkamp.

Bourdieu, P. (2000). Die zwei Gesichter der Arbeit. Interdependenzen von Zeit- und Wirtschaftsstrukturen am Beispiel einer Ethnologie der algerischen Übergangsgesellschaft. Konstanz: UVK. 
Bourdieu, P. (2007). Die feinen Unterschiede. Kritik der gesellschaftlichen Urteilskraft. Frankfurt a. M.: Suhrkamp.

Bourdieu, P., \& Boltanski, L. (1975). Le titre et le poste: Rapports entre le système de production et le système de reproduction. Actes de la recherche en sciences sociales, 1, 95-107.

Bourdieu, P., \& Wacquant, L. J. D. (1996). Reflexive Anthropologie. Frankfurt a. M.: Suhrkamp.

Bourdieu, P., Boltanski, L., \& Chamboredon, J.-C. (1963). La Banque et sa clientèle. Éléments d'une sociologie du crédit. Paris: Centre de sociologie européenne de l'École Pratique des Hautes Études.

Boyer, R. (2003). L'anthropologie économique de Pierre Bourdieu. Actes de la recherche en sciences sociales, 150, 65-78.

Costa, R. L. (2006). The logic of practices in Pierre Bourdieu. Current Sociology, 54, 873-895.

Deutschmann, C. (2007). Unsicherheit und soziale Einbettung: Konzeptionelle Probleme der Wirtschaftssoziologie. In J. Beckert, R. Diaz-Bone, \& H. Ganßmann (Hrsg.), Märkte als soziale Strukturen (S. 79-93). Frankfurt a. M.: Campus.

Diaz-Bone, R. (2007). Qualitätskonventionen in ökonomischen Feldern. Berliner Journal für Soziologie, 17, 489-509.

Diaz-Bone, R. (2015). Die „Economie des conventions“. Grundlagen und Entwicklungen der neuen französischen Wirtschaftssoziologie. Wiesbaden: Springer VS.

Dobbin, F. (2004). The new economic sociology. A reader. Princeton: Princeton University Press.

Eloire, F. (2014). The effects of social capital on economic and symbolic profits. An analysis of the field and networks of restaurant owners. In A. Christoforou \& M. Lainé (Hrsg.), Re-thinking economics. Exploring the work of Pierre Bourdieu (174-191). New York: Routledge.

Fligstein, N. (1981). Going north. Migration of blacks and whites from the South, 1900-1950. New York: Academic Press.

Fligstein, N. (1990). The transformation of corporate control. Cambridge: Harvard University Press.

Fligstein, N. (1997). Social skill and institutional theory. American Behavioral Scientist, 40, $397-405$.

Fligstein, N. (2010). Die Architektur der Märkte. Wiesbaden: VS Verlag für Sozialwissenschaften.

Fligstein, N., \& Goldstein, A. (2015). The emergence of a finance culture in American households, 1989-2007. Socio-Economic Review, 13, 575-601.

Fligstein, N., \& McAdam, D. (2011). Toward a general theory of strategic action fields. Sociological Theo$r y, 29,1-26$.

Fligstein, N., \& McAdam, D. (2012a). A theory of fields. New York: Oxford University Press.

Fligstein, N., \& McAdam, D. (2012b). Response to Goldstone and Useem. Sociological Theory, 30, 48-50.

Fligstein, N., \& Stone Sweet, A. (2002). Constructing polities and markets: An institutionalist account of European integration. American Journal of Sociology, 107, 1206-1243.

Florian, M. (2008). Felder und Institutionen. Der soziologische Neo-Institutionalismus und die Perspektiven einer praxistheoretischen Institutionenanalyse. Berliner Journal für Soziologie, 18, 129-155.

Fourcade, M. (2013). Wirtschaftssoziologie und Gesellschaftstheorie. In J. Maeße (Hrsg.), Ökonomie, Diskurs, Regierung (S. 35-56). Wiesbaden: Springer VS.

Granovetter, M. (1985). Economic action and social structure: The problem of embeddedness. American Journal of Sociology, 91, 481-510.

Kluttz, D. N., \& Fligstein, N. (2016). Varieties of sociological field theory. In S. Abrutyn, (Hrsg.), Handbook of Contemporary Sociological Theory (S. 185-204). Wiesbaden: Springer VS.

Krippner, G. R., \& Alvarez, A. S. (2007). Embeddedness and the intellectual projects of economic sociology. Annual Review of Sociology, 33, 219-240.

Lamont, M. (2012). Toward a comparative sociology of valuation and evaluation. Annual Review of Sociology, 38, 201-221.

Lebaron, F. (2003). Pierre Bourdieu: Economic models against economism. Theory and Society, 32, $551-565$.

Lebaron, F. (2009). How Bourdieu "quantified” Bourdieu: The geometric modelling of data. In K. Robson \& C. Sanders (Hrsg.), Quantifying Theory: Pierre Bourdieu (S. 11-29). Dordrecht: Springer.

Lewin, K. (1939). Field theory and experiment in social psychology: Concepts and methods. American Journal of Sociology, 44, 868-896.

Martin, J. L. (2003). What is field theory? American Journal of Sociology, 109, 1-49.

Maurer, A. (2006). Wirtschaftssoziologie als soziologische Analyse ökonomischer Felder? Bourdieus Beitrag zur Wirtschaftssoziologie. In M. Florian \& F. Hillebrandt (Hrsg.), Pierre Bourdieu: Neue Perspektiven für die Soziologie der Wirtschaft (S. 127-146). Wiesbaden: VS Verlag für Sozialwissenschaften.

McAdam, D. (1990). Freedom summer. Oxford University Press. 
McAdam, D. (1999). Political process and the development of black insurgency, 1930-1970. Chicago: University of Chicago Press.

Medvetz, T. (2013). Les think tanks dans le champ du pouvoir étasunien. Actes de la recherche en sciences sociales, 200, 44-55.

Müller, H.-P. (2014). Pierre Bourdieu: Eine systematische Einführung. Berlin: Suhrkamp.

Mützel, S. (2006). Strukturelle Netzwerkanalyse und Bourdieus Praxistheorie: Weiterführende Ideen für die neue Wirtschaftssoziologie. In M. Florian \& F. Hillebrandt (Hrsg.), Pierre Bourdieu: Neue Perspektiven für die Soziologie der Wirtschaft (S. 109-125). Wiesbaden: VS Verlag für Sozialwissenschaften.

Mützel, S. (2009). Networks as culturally constituted processes. A comparison of relational sociology and actor-network theory. Current Sociology, 57, 871-887.

Powell, W. W., \& DiMaggio, P. J. (1983). The iron cage revisited: Institutional isomorphism and collective rationality in organizational fields. American Sociological Review, 48, 147-160.

Schmitz, A. (2016). The structure of digital partner choice. New York: Springer.

Schmitz, A., Witte D., \& Gengnagel V. (2016). Pluralizing field analysis: Toward a relational understanding of the field of power. Social Science Information, 56, 49-73.

Schultheis, F. (2007). Bourdieus Wege in die Soziologie: Genese und Dynamik einer reflexiven Sozialwissenschaft. Konstanz: UVK.

Sewell, W. H. (1992). A theory of structure: Duality, agency, and transformation. American Journal of Sociology, 98, 1-29.

Sparsam, J. (2015). Wirtschaft in der New Economic Sociology. Eine Systematisierung und Kritik. Wiesbaden: Springer VS.

Suckert, L. (2015). Die Dynamik ökologischer Märkte: Eine feldanalytische Betrachtung des Marktes für Bio-Molkereiprodukte. Konstanz: UVK.

Suckert, L (2017). Unravelling Ambivalence: A field-theoretical approach to moralised markets. Current Sociology. Online first 20. November 2017, https://doi.org/10.1177/0011392117737820.

Swartz, D. L. (2014). Theorizing fields. Theory and Society, 43, 675-682.

Swedberg, R. (2009). Grundlagen der Wirtschaftssoziologie. Wiesbaden: VS Verlag für Sozialwissenschaften.

Thévenot, L. (2007). The plurality of cognitive formats and engagements: Moving between the familiar and the public. European Journal of Social Theory, 10, 409-423.

Weber, M. (1904). Die „Objektivität“ sozialwissenschaftlicher und sozialpolitischer Erkenntnis. Archiv für Sozialwissenschaft und Sozialpolitik, 19(1), 22-87.

Witte, D. (2014). Auf den Spuren der Klassiker. Pierre Bourdieus Feldtheorie und die Gründerväter der Soziologie. Konstanz: UVK.

Zelizer, V. A. (2012). How I became a relational economic sociologist and what does that mean? Politics \& Society, 40, 145-174.

Zukin, S., \& DiMaggio, P. (1990). Structures of capital: the social organization of the economy. Cambridge: Cambridge University Press.

Lisa Suckert geb. 1983. Dr. rer. Pol., Wissenschaftliche Mitarbeiterin am Max-Plack-Institut für Gesellschaftsforschung. Forschungsschwerpunkte: Wirtschaftssoziologie, Soziologische Theorie, Kapitalismus und Zukunftsvorstellungen, Konventionen und Diskurse, Nachhaltiges Wirtschaften. Ausgewählte Veröffentlichungen: Vom Konkurrenten zum Untersuchungsgegenstand: Die Wirtschaftswissenschaften im Fokus (wirtschafts-) soziologischer Kritik, in: Soziologische Revue, 2016; Die Dynamik ökologischer Märkte: Eine feldanalytische Betrachtung des Marktes für Bio-Molkereiprodukte, 2015; Organisierter Kompromiss: Wie Ecopreneur-Unternehmen das Dilemma der Nachhaltigkeit lösen, in: L. Knoll (Hrsg.) Organisationen und Konventionen, 2014. 